\title{
NOTES
}

\section{ANOVA, Latin square, and ANOCOVA for IBM-compatible microcomputer systems}

\author{
ALLEN H. WOLACH \\ Illinois Institute of Technology, Chicago, Illinois
}

Analysis of variance programs and related programs (e.g., $t$ tests, Latin squares, and analyses of covariance) are available in a comprehensive package for IBMcompatible microcomputer systems. The programs handle up to three factors, with any number of levels for a given factor. Independent groups, split-plot, and randomized block designs are included. Levels of independent group factors do not require equal numbers of subjects. Difference formulas and provisional mean formulas are used to produce accurate results across a wide range of data sets.

Description. The following 14 programs are included:

1. An independent groups $t$ test that does not require equal numbers of observations for the two groups.

2. A correlated measures $t$ test that requires equal numbers of observations for both treatments.

3. An independent groups one-way analysis of variance (completely randomized $K$-group design) that does not require equal numbers of observations for each group.

4. A randomized block $K$-treatment design that requires equal numbers of observations for each treatment.

5. A randomized block $K$-treatment design that does not require equal numbers of observations for each treatment (Yates's, 1933, procedure for estimating missing scores is used).

6. An independent groups two-way analysis of variance (completely randomized $J \times K$ design) that does not require equal numbers of observations for the subgroups.

7. A split-plot factorial $J \times K$ design that does not require equal numbers of observations for the independent groups factor.

8. A randomized block factorial $J \times K$ design that requires equal numbers of observations in all cells.

9. An independent groups three-way analysis of variance (completely randomized $J \times K$ design) that does not require equal numbers of observations for the subgroups.

10. A split-plot factorial $J \times K \times L$ design ( $L$ is the repeated measures factor) that does not require equal numbers of observations for independent groups factors $J$ and $K$.

11. A split-plot factorial $J \times K \times L$ design ( $K$ and $L$ are the repeated measures factors) that does not require an

Address correspondence to Allen H. Wolach, Department of Psychology, Illinois Institute of Technology, Chicago, IL 60616. equal number of observations for independent groups factor $J$.

12. A randomized block factorial $J \times K \times L$ design that requires the same number of observations in all cells.

13. A Latin square design that requires an equal number of observations in all cells.

14. A one-factor analysis of covariance that requires an equal number of observations in all cells.

All programs print the mean values for follow-up tests. Variances are provided for the smallest unit (group, treatment, cell, or subgroup) in each analysis. $F$ ratios are presented under the assumption that all factors except subjects are fixed effects factors (Wolach, 1983). Because all sums of squares and mean squares are presented, it is very easy to calculate $F$ ratios for random and mixed models (Wolach \& McHale, 1987).

The 157-page documentation and programs are on two 5.25-in. disks, from which they can be printed. The user must first obtain the A prompt from DOS. The documentation, in chapters, is printed by entering the word TYPE, followed by a space, followed by the file name for a chapter, followed by depression of the Control and the Print Screen keys, followed by a depression of the Enter key. Each of the 14 statistics has a complete chapter in the book to explain data entry and interpretation of output. The last chapter provides the specific formulas that were used in the programs. File names for the chapters are BEGIN, CHAP01, CHAP02, ..., CHAP15, CHAP15A, and REFANDIN. BEGIN and chapters 1 through 12 are on Disk 1. Chapters 13, 14, 15, 15A, and REFANDIN are on Disk 2. Disk 2 also contains the 14 statistical programs.

Each disk contains an AUTOEXEC.BAT file that can be used to print the book chapters without stopping after every chapter. The entire book can be printed by obtaining the A prompt, typing AUTOEXEC, followed by depression of the Control and Print Screen keys, and then by depression of the Enter key.

The user must have an interpreter version of BASIC (BASIC, BASICA, Microsoft BASIC, or GWBASIC) to use the programs. After the A prompt is obtained from DOS, the BASIC interpreter is loaded into the computer. Then the user follows the appropriate example and explanation of data entry.

Input. The book describes data entry procedures for all 14 programs. The programs were written to maintain a consistent data entry procedure from program to program. Data are entered in DATA statements that can be saved on a disk for use with other programs. Each chapter in the book includes a sample set of data and an example of data entry for the sample data. 
Output. The programs provide output to the monitor screen. If the user depresses the Control and Print Screen keys, output is sent to the printer. The book contains an example of output for each of the data sets used to demonstrate data entry.

Availability. The programs and text are available on two disks for IBM-compatible computers. Send two blank disks with the request, or $\$ 2$ for disks that will be supplied. Printed copies of the book are available for $\$ 12$. Requests for copies of the programs, the disks, and/or the book should be sent to the author.

\section{REFERENCES}

WoLACH, A. H. (1983). BASIC analysis of variance programs for microcomputers. Monterey, CA: Brooks/Cole.

Wolach, A. H., \& McHAle, M. A. (1987). $F$ ratios and quasi $F$ ratios for fixed, mixed, and random model ANOVAs. Behavior Research Methods, Instruments, \& Computers, 19, 409-412.

YATES, F. (1933). The analysis of replicated experiments when field results are incomplete. Empire Journal of Experimental Agriculture, 1, 129-142.

(Revision accepted for publication October 15, 1988.) 